\title{
Performing social media analytics with Brandwatch for Classrooms: a platform review
}

\author{
Mike McGuirk ${ }^{1}$
}

Revised: 15 July 2021 / Accepted: 21 July 2021 / Published online: 19 August 2021

(c) The Author(s), under exclusive licence to Springer Nature Limited 2021

\begin{abstract}
As consumer's consumption of digital media and usage of social media platforms continues to grow, businesses have been searching for new ways to track, analyze, and measure this online consumer behavior to support a diverse set of business functions such as marketing, customer support, and customer experience management. Given the heightened interest in social media analytics in the business community, educational institutions have also been interested in identifying new ways to introduce emerging social media analytics concepts and practices to their students. One category of social technologies that is specifically used to perform this type of analysis is social listening platforms. Brandwatch (recently acquired by Cision Ltd.) has created one of the leading social listening platforms. They also offer a version of this platform for educators named Brandwatch for Classrooms. This article provides an overview of the key features and capabilities of the Brandwatch for Classrooms platform, as well as numerous suggestions for incorporating the platform in marketing analytics undergraduate and graduate courses. The goal is to provide educators with practical information about social media analytics and guidance on successfully integrating social listening platforms in their course design.
\end{abstract}

\section{Introduction}

It is important to provide the proper context for the review of Brandwatch for Classrooms by first describing the current state of social media usage globally. This is followed by a description of the different types of social media data and the social media marketing technologies used to collect and analyze the data. The introduction will conclude with a summary of the primary social media analytics techniques and most popular marketing-oriented applications of the social media analytics insights.

This contextual information will be utilized to develop a social media analytics framework that is suitable for reviewing the capabilities of the Brandwatch for Classrooms product relative to the business needs and the standards that have been established for collecting and analyzing social media data.

Mike McGuirk

mmcguirk@babson.edu

1 Marketing Division, Babson College, Babson Park, MA 02457, USA

\section{Social media usage}

As of April 2021, 4.33 billion people or $55.1 \%$ of the global population was actively using social media. That represents a $13.7 \%$ increase over the last 12 months (DataReportal 2021). This includes usage across a variety of social media platforms such as social networks, question and answer sites, forums and message boards, messaging and live chat services, micro-blogs, vlogs, online pinboards, review sites, wikis, and content communities. Facebook has the most active users ( 2.8 billion), followed by YouTube ( 2.3 billion) and WhatsApp ( 2 billion). The active user counts for other popular platforms are Facebook Messenger (1.3 billion), Instagram (1.3 billion), TikTok (732 million), Snapchat (528 million), Pinterest (459 million), Twitter (396 million), and Quora with 300 million active users (DataReportal 2021).

Geographically, based on January 2021 statistics, Western and Northern Europe had the highest social media penetration rate of $79 \%$, with Northern America (74\% penetration) following close behind. Southeastern Asia and Eastern Asia had penetration rates of $69 \%$ and $66 \%$, respectively. In contrast, Southern Asia (31\% penetration), Western Africa (16\% penetration), and Eastern Africa (10\% penetration) had significantly lower social media penetration (Statista 2021). 
Of particular importance given the marketing analytics focus of this article, $72.1 \%$ of active social media users use at least one type of social media platform to research brands. Social networks is the most popular platform used for researching brands ( $44.7 \%$ of active users), followed by question and answer sites (20.8\% of active users), and forums and message boards representing $16.7 \%$ of active users (DataReportal 2021).

Not only is social media penetration increasing across most parts of the world, the average time spent on social media platforms is also increasing at a dramatic rate. In 2012 , the average daily time spent on social media was 90 min per day. That has increased by $58 \%$, and in 2021 the average daily time spent on social media is $142 \mathrm{~min}$ per day (DataReportal 2021; Statista 2021).

Given the rapid growth in social media usage rates, it is not surprising that customer-centric organizations are eager to harvest the social conversion data generated by consumers and businesses to help inform their marketing strategies. The subsequent section describes the type of data that is available across social media sources.

\section{Social media data}

Social media data can be generated from many different sources and stored in many different formats. Borrowing from the terminology used to describe big data, it would be fair to claim that social media data check the boxes of all four requirements of big data. That is, an extremely high Volume of data can be produced with great Velocity, containing a Variety of formats with sometimes uncertain Veracity (Stieglitz et al. 2018; McAfee and Brynjolfsson 2012). These data can be "consumer-generated" (e.g., comments, posts, tweets, inquiries, reviews, images, audio, video), "firm-generated" (e.g., webpages, product descriptions, posts, comments, images, audio, video), "platformgenerated" (e.g., followers, likes, shares, ratings, stars, rankings), and "network-generated" via links between social media users (Wang et al. 2021; Calheiros et al. 2017; Felbermayr and Nanopoulos 2016; Moon and Kamakura 2017; Alp and Öğüdücü 2018; Kumar et al. 2016).

Most social media data are stored in either a structured or unstructured format. Structured data adhere to well-defined, standardized data formats, whereas unstructured data are generally more challenging to process because the format is not pre-defined, such as a Facebook post (Hartman 2020). Multi-lingual text, video, and audio data are examples of unstructured data sources. Extracting business intelligence from these sources generally requires the use of advanced rules-based or machine learning-based natural language processing tools and techniques (Khan 2018; SAS 2020). It is currently estimated that eighty percent of all data collected by organizations is stored in an unstructured format (Hartman 2020).

The social marketing technologies discussed in the next section are commonly used to enable the large-scale collection, data transformation, and analysis of social media data.

\section{Social media marketing technologies}

Several different social technologies are utilized to meet the needs of social marketers. Social technologies can be divided into three primary categories: "social listening platforms," "social advertising technology," and "social suites" (Liu and Dawson 2021). Social listening platforms, the focus of this article, are used to collect, manage, and analyze social media data. Social adtech is used to manage and measure the advertising on social media sources. Social suites include many of the capabilities of separate social technologies into one platform. They are used to perform tasks such as data collection and analysis, publishing content, and managing customer communications (Liu and Dawson 2021).

It is important to note that social listening platform (SLP) providers are in a "transitional" state. For example, providers are determined to deliver on marketers' entire digital customer intelligence needs, moving beyond the traditional bounds of social media data providers. One of the significant enhancements SLPs will be required to embed in future solutions is the ability to integrate proprietary customer data (e.g., customer tenure, customer spend, products purchased) in addition to the social media data that is already captured (Liu and Dawson 2020). This enhanced dataset will help organizations move towards an assessment of the business impact of social marketing strategies and programs, versus an assessment of specific content or marketing campaigns (Liu 2021).

The "Forrester Wave ${ }^{\mathrm{TM}}$ : Social Listening Platforms, Q4 2020 " report provides a comprehensive evaluation of the top SLP providers in the market. The report separates these providers into leaders, strong contenders, contenders, and challengers based on their current offering, strategy, and market presence. The Brandwatch Consumer Research platform was evaluated along with nine other SLP providers (Digimind, Linkfluence, ListenFirst, Meltwater, NetBase Quid, Sprinklr, Synthesio, Talkwalker, and Zignal Labs) in the most recent report. The Brandwatch platform was named a leader in the SLP market and had the highest scores in the current offering, strategy, and market presence categories (Liu and Dawson 2020).

Please note that the Brandwatch for Classrooms product will be reviewed in this article. This product shares almost all of the same features and capabilities as the Brandwatch Consumer Research platform reviewed in the Forrester Wave $^{\mathrm{TM}}$ report; however, it has some licensing and usage 
agreement restrictions that will be highlighted later in the product review.

\section{Social media analytics}

The growth in the usage of social media platforms and the advancements in social listening platform capabilities have enabled the widespread adoption of many different types of social media analytics (SMA) that can be performed by marketing professionals with even limited experience in analytics.

SMA is the application of data management and analytics tools and techniques to "collect, monitor, analyze, summarize, and visualize" social media data to uncover useful information, trends and behaviors (Zeng et al. 2010; Zhan et al. 2021). This often includes the analysis of unstructured and structured social media data across a wide variety of digital channels to examine how individuals and businesses "think and feel" about a wide array of topics (Wamba et al. 2016; Chen et al. 2012).

The SMA process is commonly separated into four primary distinct steps, including "Discovery, Tracking, Preparation, and Analysis" (Stieglitz et al. 2018).

- Discovery: identification of content and corresponding keywords, hashtags, images, etc. used when discussing specific topic(s) that will provide input into framing the analysis objectives and the primary hypotheses to be tested.

- Tracking: determination of the data sources, collection approach, and output of data on the discovered topic.

- Preparation: the approaches (e.g., natural language processing, topic categorization) used to prepare the source data for subsequent analysis.

- Analysis: conducting various analysis methods/techniques on the prepared data set that aim to answer the questions outlined in the discovery phase.

The analysis stage can include many different types of SMA techniques. "Text mining, sentiment analysis, network analysis, and image analytics" are four common SMA techniques deployed by organizations (Wang et al. 2021).
Text mining refers to "content-based analysis" (Gandomi and Haider 2015). This includes techniques such as "keyword retrieval, topic isolation, topic modeling, and cluster analysis" (Felbermayr and Nanopoulos 2016; Nam et al. 2017). Sentiment analysis also leverages text mining techniques with the goal of classifying social media data/content by the sentiment and emotion of the social media content author (Calheiros et al. 2017). The most common type of network analysis using social media data is "social network analysis," which examines the relationship between "human networks" (Wang et al 2021; Ikeda et al. 2013). Image analytics is an emerging SMA field of study, which includes the analysis and classification of images and visual content (Miah et al. 2017). The development of deep learning models has become a popular approach to perform highly sophisticated image analytics (Shin et al. 2020). The deep learning models can "generate generic image features in a scalable manner" that allow analysts to assess the impact of specific images on social media programs (Shin et al. 2020).

\section{Social media analytics use cases}

Social media analytics are performed to provide insights that support many different business functions and business needs. Several use cases that are relevant to marketing and related business functions are provided in Fig. 1 (Brandwatch, 2021a; Wang et al 2021).

In general, the overarching objective of these use cases is to leverage the social media analytics insights to apply a data-driven approach to inform the "product, marketing, and overall business strategy" decision making (Liu and Parrish 2020).

Many social listening platforms are also designed to promote "data democratization" by developing user interfaces and data analysis tools that can be operated by employees with very limited knowledge in marketing analytics (Brandwatch, 2021a; LeClair 2018).

In addition, several SLP vendors are beginning to develop solutions to augment social listening data with enterprise data such as sales, revenue, and customer loyalty to enable the measurement of social media strategies and the impact they have on business value (Liu 2021).
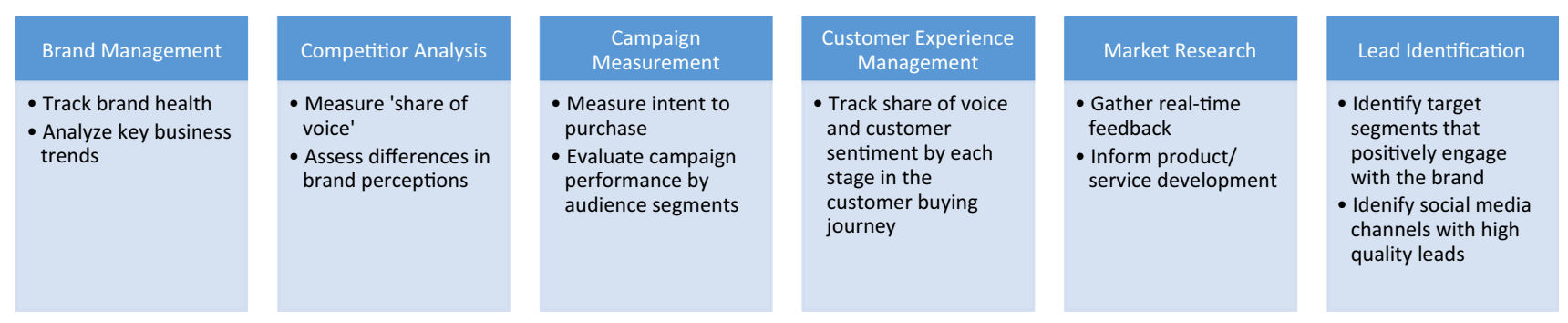

Fig. 1 Social media analytics use cases in marketing-oriented business functions 


\section{Brandwatch for Classrooms product review}

\section{Brief orientation}

Brandwatch for Classrooms is one of several educational products offered by Brandwatch. It is designed specifically for educators and researchers. The current product can be licensed for \$1500USD per year (Brandwatch 2021b). It provides access to the Brandwatch Consumer Research enterprise platform, but the licensing agreement does include some usage and data restrictions, including a limit of ten queries at a time, a download limit of up to 100k mentions per day, and access of up to two years of historical data. Queries and mentions will be defined later in this section.

The four-step process for performing SMA that was introduced in the prior section will be utilized as a framework to describe the core features and tools available in the Brandwatch platform to perform each of these steps. However, the framework has been extended to also include a "deployment" step. The deployment phase addresses the need to effectively communicate and transform the SMA results into relevant business insights and data-driven business actions. The final phase label, deployment, was inspired and adopted from the final step in the Cross Industry Standard Process for Data Mining, abbreviated as CRISP-DM (Jaggia et al. 2020).

Please refer to Fig. 2 for a mapping of core Brandwatch features/tools to each step in the SMA process. The labels in parentheses indicate the higher-level category where these features are located in the Brandwatch platform user interface. Please note that this mapping is not intended to be an exhaustive listing of the Brandwatch platform features; however, they represent many of the core features used by marketers.

The Brandwatch platform user interface incorporates navigational tabs, each containing multiple features and/ or tools that support the five SMA process steps. Users can also access many Help and Support resources such as training videos, how-to-guides, use cases, community discussion boards, and detailed documentation describing how to use each of the platform features. Figure 3 contains a partial image of the Home page on the Brandwatch platform (Brandwatch 2021c).

The Home page also displays alerts and important trends using the Brandwatch AI-powered analyst assistant called Iris. Iris is designed to automatically analyze peaks in the social media data and helps detect what caused social conversations to spike (Brandwatch 2021d).

Brandwatch uses the term mentions to describe a piece of "web-based content posted to or from a social channel" (Brandwatch 2021d). For example, this content could represent a tweet or a caption on an uploaded image on Instagram. This term will be used throughout the article.

\section{Brandwatch discovery features}

A common challenge when conducting exploratory SMA analysis is that the "topics might not be known a priori, and have to be discovered first" (Stieglitz et al. 2018). This is often the case in marketing applications of SMA, when the analyst is interested in discovering new insights related to recent customer experiences with a brand.

Brandwatch addresses this challenge by providing a user-friendly search feature (Brandwatch Search) that is designed to easily and quickly return nineteen pre-defined data visualizations/statistics on any brand, people, or topics to determine if this is something the analyst would like to more thoroughly analyze using other platform features (Brandwatch 2021e).

Brandwatch Search is an AI-based search feature that utilizes sophisticated natural language processing (NLP) techniques. Simply put, NLP is a branch of artificial intelligence that is used to discover meaning in human language (Hair Jr. et al. 2021). Brandwatch Search allows the user to type in a search term(s), and it uses three natural language processing steps and a language model to immediately retrieve mentions that match the search request (Brandwatch 2021f). Figure 4, sourced by Brandwatch, illustrates how this three-step process operates on a sample Twitter tweet (Brandwatch 2021f).

1. Noun-phrase extraction-extracts important noun phrases from the Brandwatch mentions library

2. Entity candidate generation-identifies possible candidate definitions for each noun-phrase (entity)
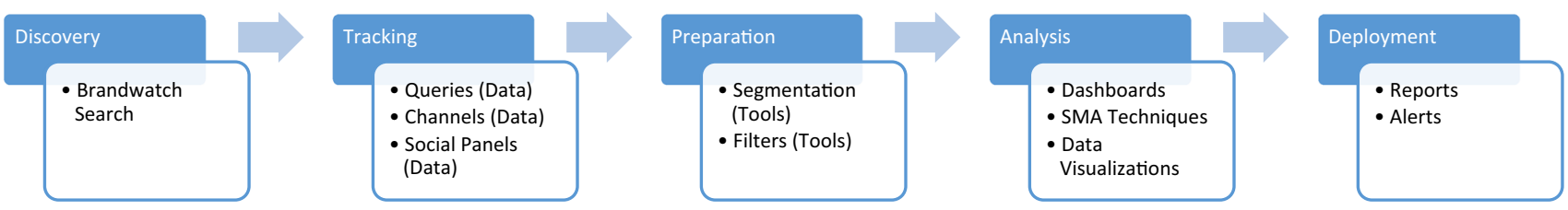

Fig. 2 Mapping of core Brandwatch features to the five-step modified SMA process framework

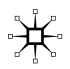




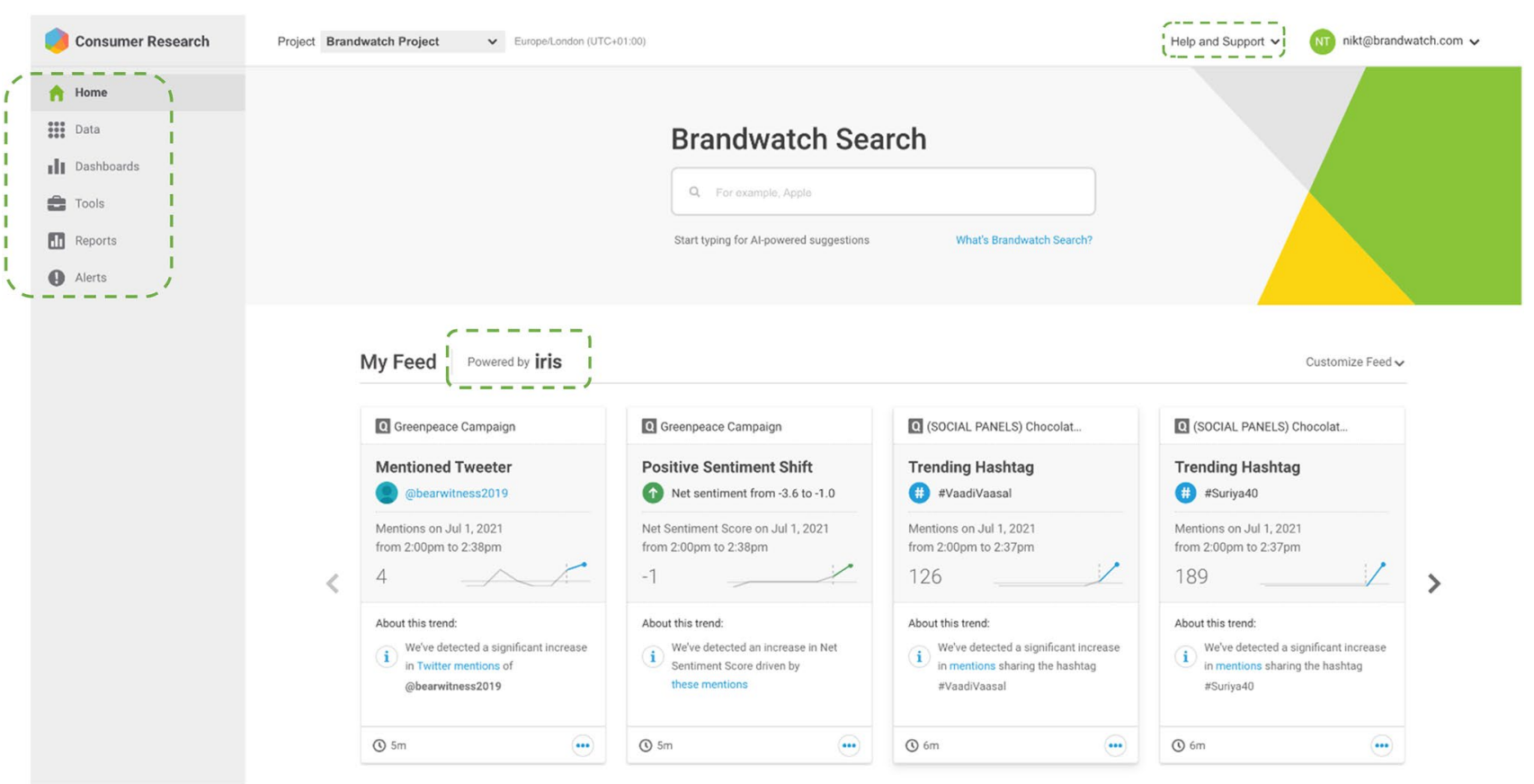

Fig. 3 Brandwatch user interface (partial view of the home page)

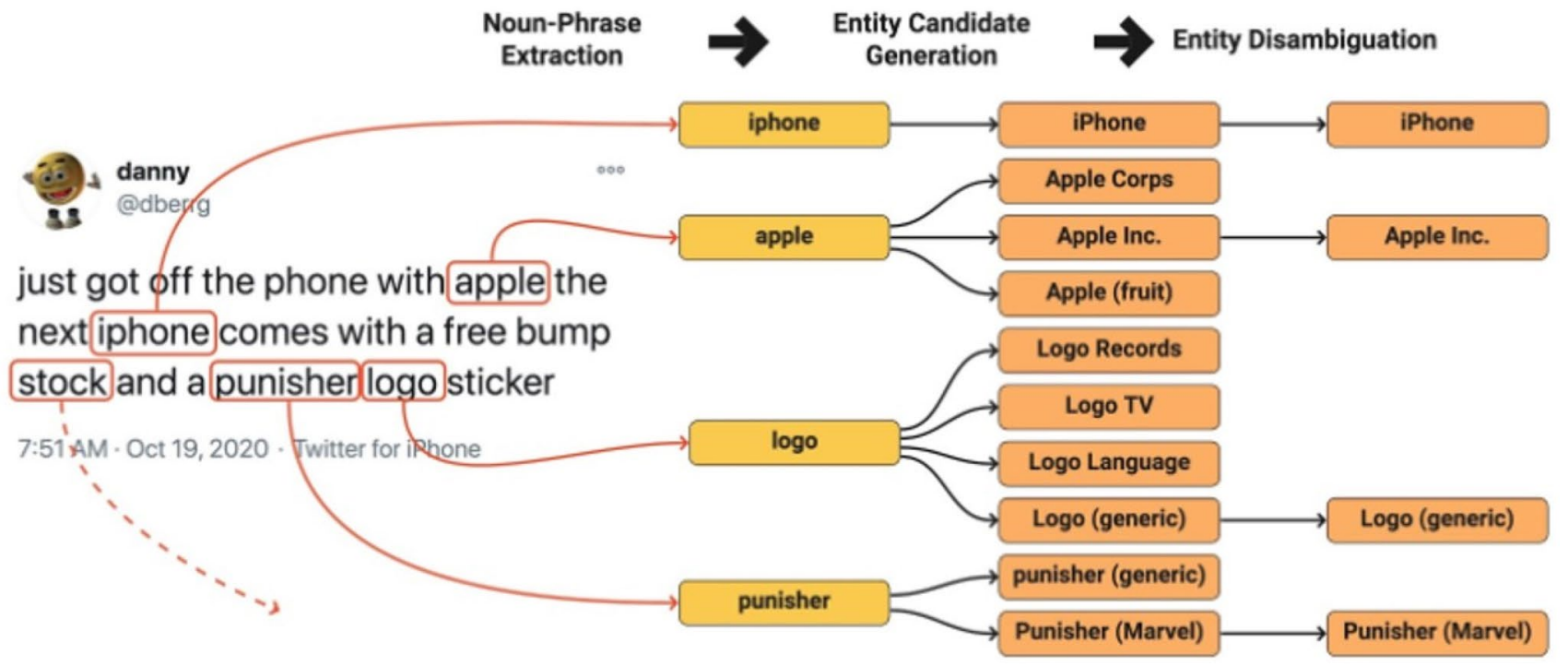

Fig. 4 Three-step entity extraction process used in Brandwatch search process

3. Entity disambiguation-determines which entity candidate is most likely being used in the mention

All of these complex NLP sub-steps as well as the search term matching to social media content happens instantly behind the scenes, allowing users with no analytic expertise to perform and complete the discovery phase. In fact, this desire for "data democratization" by making the Brandwatch platform accessible to users with all different levels of analytic expertise is achieved at each step in the SMA process through the integration of user-friendly tools (LeClair 2018).

\section{Brandwatch tracking features}

Brandwatch Queries is a core feature that is used to initially collect and continuously refresh the data you would like to capture for subsequent analysis. The data that are 
being collected are often referred to as "social big data" (Guellil and Boukhalfa 2015) or "social media big data" (Lynn et al. 2015) and adhere to the 4V's defined earlier in the article. This type of data poses several challenges (Khan 2018; Stieglitz et al. 2018) that the Brandwatch platform effectively addresses (Brandwatch 2021p). Table 1 lists the challenges and corresponding solutions.

The queries editor allows the user to write query logic that utilizes Boolean operators, pre-built text string functions, filters, and 152 language options to precisely target and retrieve the types of mentions you want to analyze. In addition, you can retrieve data based on searching for occurrences of logos and images in the social media data, or even retrieve data related to a group of targeted social media authors using social panels (Brandwatch 2021g). The queries editor also provides a testing preview window to instantly evaluate the type of mentions being retrieved from the current query logic. This allows the user to adjust and fine tune the precision of the query logic to return only the types of mentions that meet your analysis needs.

In Brandwatch for Classrooms, once the query is saved, you can use it to collect historical information dating back two years, and the query will also continue to collect new mentions in near real-time moving forward. If needed, Brandwatch provides a built-in random sampling approach to address queries that would retrieve extremely large amounts of mentions. The sampling rate will be automatically set to ensure the data collection does not exceed the license agreement.

Brandwatch Channels is another data tracking tool, and it provides the ability to collect and track data from owned and non-owned social media channels. The level of tracking is specific to the social media channel being tracked as well as the owner/non-owner status of that channel. Currently, Brandwatch for Classrooms users can set up channel tracking on Facebook, Instagram, and Twitter channels. The current process to set up Facebook and Instagram channels is quite cumbersome. However, the Brandwatch 2021 roadmap highlights the company's desire to enhance the channel tracking for Facebook and Instagram, by allowing the collection of more historical data and by simplifying the UX design to make it easier to set up the collection of data from these sources (Brandwatch 2021h). The license limits data collection to 10 Twitter channels and 10 combined Facebook/Instagram channels.

Brandwatch Social Panels is a relatively new feature in the platform, and it allows users to create an online opinion research panel as an alternative way to collect social media data. You can construct social panels in two different ways (Brandwatch 2021i):

1. Upload a list of online social media authors (handles) that you want to include in the panel. These can be authors on any social media platforms.

2. Use the Brandwatch platform search capability to identify authors to include in the social panel (currently limited to Twitter authors). Several types of filters can be used to identify potential panelists, such as the authors influence score, number of followers, bio keywords, demographics, interests, and geography. Currently, the platform only allows selection of panelists using the search functionality from the Twitter social media channel. Brandwatch is in the process of updating this feature to allow selection of panelists using the search functionality from additional social media channels.

\section{Brandwatch preparation features}

Social media data require pre-processing and data preparation steps to fully harvest the consumer and business insights. As stated earlier, the data are largely unstructured and are prone to many types of issues such as abbreviations, ambiguous language, misspellings, grammatical errors, and a wide array of different data formats (Rehman et al. 2016).

For the most part, the discovery and tracking of this unstructured data are managed by the Brandwatch search and queries capabilities. However, additional tools are needed to manage the structuring of the raw data in customized ways that meet the needs of the analysis.

That is, social listening platforms need to provide tools that help the analyst categorize the social media data into meaningful segments. The segmentation tools should allow the user to easily divide unstructured consumer mentions and posts into unique categories. This type of content/data

Table 1 Social big data collection challenges and Brandwatch solutions

\begin{tabular}{|c|c|}
\hline Challenge & Brandwatch solution \\
\hline Managing large storage requirements (volume) & $\begin{array}{l}\text { Sampling techniques are applied to remain within the } 100 \mathrm{k} \text { mention limit per day without } \\
\text { sacrificing the representativeness of the data }\end{array}$ \\
\hline Enabling fast ingestion of new data (velocity) & Data retrieved by queries is refreshed in near real-time \\
\hline $\begin{array}{l}\text { Accurately processing structured and unstruc- } \\
\text { tured data sources (variety) }\end{array}$ & Sophisticated NLP and data classification tools are utilized \\
\hline $\begin{array}{l}\text { Handling data sources with uncertain levels of } \\
\text { quality (veracity) }\end{array}$ & $\begin{array}{l}\text { Spam-filters and intelligent data crawlers are utilized to reduce noise and track relevant conver- } \\
\text { sations }\end{array}$ \\
\hline
\end{tabular}


segmentation can encompass a broad set of dimensions such as geography, topics, content sources, consumer sentiment and emotions, product categories, comparison of brands, stages of the consumer purchase cycle, and author demographics, interests, and professions (Jamali 2021).

The Brandwatch platform provides several pre-built segments/categories. These include categories such as consumer sentiment (negative, neutral, and positive), consumer emotions (anger, disgust, fear, joy, sadness, and surprise), content sources (blogs, forums, news, reviews, Facebook, Instagram, Reddit, Tumblr, Twitter, YouTube, etc.), images (logos, objects, scenes, actions), and several other demographics and interest categories.

The platform also provides several customizable approaches to pre-process the data and prepare it for further analysis. These include:

- The creation of rules-based categories

- The creation of machine learning-based categories

- The use of multi-dimensional filters

The Brandwatch Categories and Brandwatch Rules features are used to develop rules-based categories. The user first creates a new category dimension (e.g., product category) and then uses Boolean operators to establish the rules that map each mention into the appropriate product sub-categories.

In contrast, the Brandwatch Custom Classifiers feature utilizes a machine learning (ML) algorithm that allows the analyst to train the platform to segment data in a particular way. This is particularly useful when dealing with conversation data that are nuanced (e.g., stages of customer purchase cycle) and are difficult to categorize using a pre-defined collection of keywords. Once the ML model is trained, the algorithm will automatically assign mentions into the appropriate sub-categories (Brandwatch 2021j). This type of ML-based classification automation "extends the expert interpretation" of the social media data and facilitates largescale "knowledge management" that would be otherwise impossible (Pääkkönen et al. 2020).

Brandwatch also includes a variety of filtering features that allow the user to group together items. These include filters such as Brandwatch Site Lists, Brandwatch Author Lists, and Brandwatch Location Lists. This allows the analyst to prepare the data to later create visualizations or reports that compare and contrast different groups of sites, authors, and locations.

\section{Brandwatch analysis features}

The Brandwatch Dashboard features enable the creation of many different types of social media analysis techniques and visualizations. It includes a variety of pre-built templates that allow the user to select visualizations and descriptive statistics that meet their general needs. Furthermore, the user can use the wide array of dashboard controls to customize the dashboards and data visualizations to meet the specific needs of the analysis (Brandwatch 2021k).

The primary categories of SMA techniques, including text mining, sentiment analysis, network analysis, and image analytics (Wang et al. 2021) will be used as a framework to evaluate the Brandwatch platforms capabilities in each of these analytic areas.

The Brandwatch platform has several dashboard templates that support a variety of text mining techniques such as the Topics Wheel, Topic Cluster, Trending Topics, Word Cloud, Emoji Cloud, Twitter Themes, and Geotagged Mention Maps. Figures 5 and 6 contain two different data visualization approaches on a sample of topic data associated with electric vehicles (Brandwatch 2021c). It is important to note that most of the Brandwatch visualizations are interactive. In this example, this means that the user can click on any of the topics found in the Topic Cluster or the Word Cloud visualizations and instantly drill down to a list of the actual mentions that belong to that topic category. This allows the user to quickly gain a better understanding of the topic categories by reading through individual posts, comments, and other forms of social conversation that were collected in the Tracking phase.

The platform also provides the ability to perform thorough sentiment analysis. Because consumer sentiment and emotions are pre-built categories, the analyst can evaluate other important customized data dimensions such as "product, service, and brand" (Choi et al. 2020) through the lens of consumer sentiment and emotions categories or simply use the Brandwatch Sentiment dashboard template. For example, this type of analysis may determine that customers are generally very satisfied with the quality of a brand's core products, but they are extremely dissatisfied with the customer service responsiveness.

Network analysis in a social media context can be defined as 'identifying customers' interaction network or discovering influential users" (Choi et al. 2020). The platform does not include any out-of-the box social network graphing or mapping tools that display the nodes and relationships between social network members. However, the platform includes a comprehensive set of features that identify topic or brand influencers. The Brandwatch Influencer dashboard template provides lists of top authors, top tweeters, top bloggers, key advocates, key detractors, and influencer demographics. The template also includes useful metrics on each of the influencers such as the number of followers, their average reach, and their impact score. Reach is a proprietary algorithm that estimates the number of different individuals that viewed the influencer's mention. Impact score is a proprietary algorithm that determines the overall engagement, 

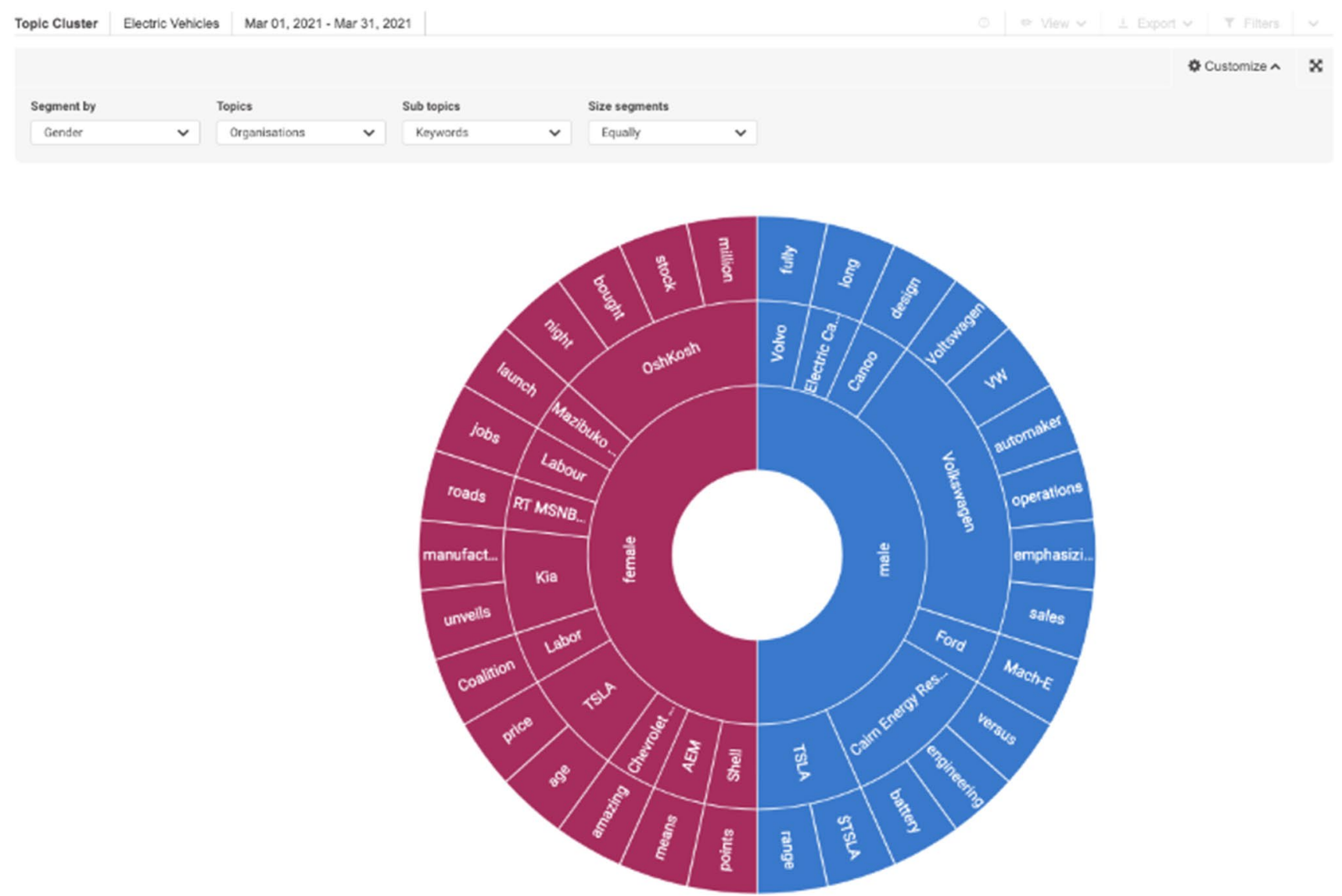

Fig. 5 Sample Brandwatch Topic Cluster By Gender, Organizations, And Keywords

on a 1-100 scale, related to each of the influencer mentions (Brandwatch 2021i).

Brandwatch also has a robust set of image analytics capabilities. Two out-of-the-box dashboard templates are available for image analysis: Brandwatch Logo Wall which displays images and corresponding captions based on the specific $\log$ o(s) that were selected in the query, and Brandwatch Image Analysis, which visually summarizes the content within the selected image data. The platform uses image recognition technology (Shin et al. 2020), which can separately identify and classify the different objects, scenes, actions, and logos appearing in images. Figure 7 provides an example of this analysis on a sample of electric vehicle topics (Brandwatch 2021c).

\section{Brandwatch deployment features}

The Brandwatch Report features make it very easy to export dashboard visualizations into Microsoft PowerPoint slides that can be used as a stand-alone document or edited to include additional analyst comments and recommendations in a project report or presentation. It is also possible to set up and generate automated reports that provide ongoing updates on a list of pre-defined social media analysis metrics that can be distributed directly to selected users email inboxes (Brandwatch 2021m).

The Brandwatch Alert features allow system detected or user-defined alerts to be sent to a specified group of individuals. For example, this could include sudden changes in negative sentiment mentions or surges in competitor brands share of voice (Brandwatch 2021n).

\section{Integrating a social listening platform into a marketing analytics course design}

Marketing analysts are increasingly asked to straddle the art and science elements of the marketing profession. That is, they need to be able to analyze social media data and convert insights into business knowledge and data-driven marketing actions (Bal et al. 2015; Kim 2019). In particular, many digital marketing analysts are expected to develop the following three skills (Hartman 2020):

- "Data strategist" skills where they "bridge the data and marketing" functions and understand how data can be 
Word Cloud Electric Vehicles Mar 01, 2021-Mar 31, 2021

Top Locations, Organizations \& People

Show Word Size

Locations, Organizati. $\checkmark$ Volume
Color

$\checkmark$ Topic Type
(1) Explore

Customizen $\mathrm{x}$

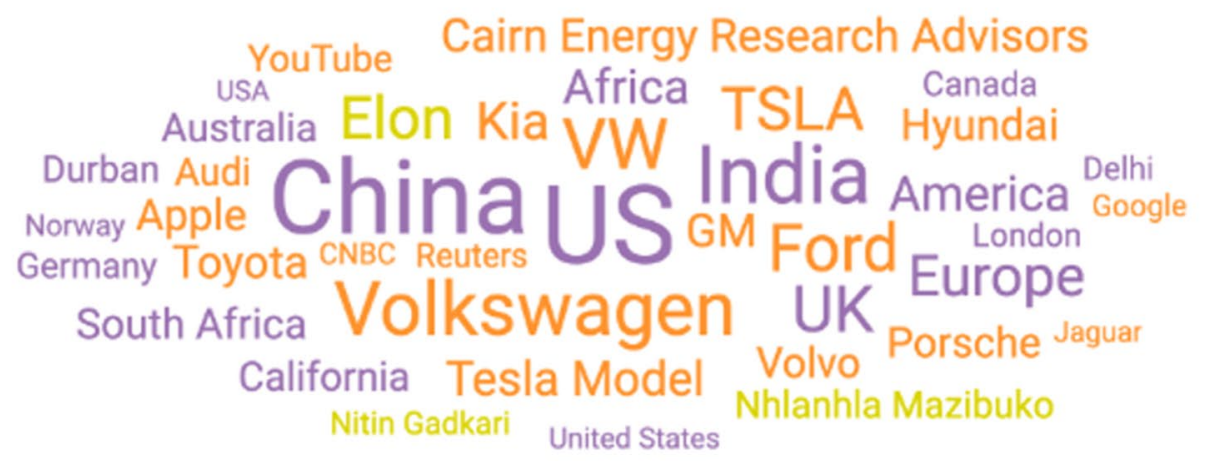

- Locations Organizations People

Fig. 6 Sample Brandwatch word cloud by pre-defined topic types

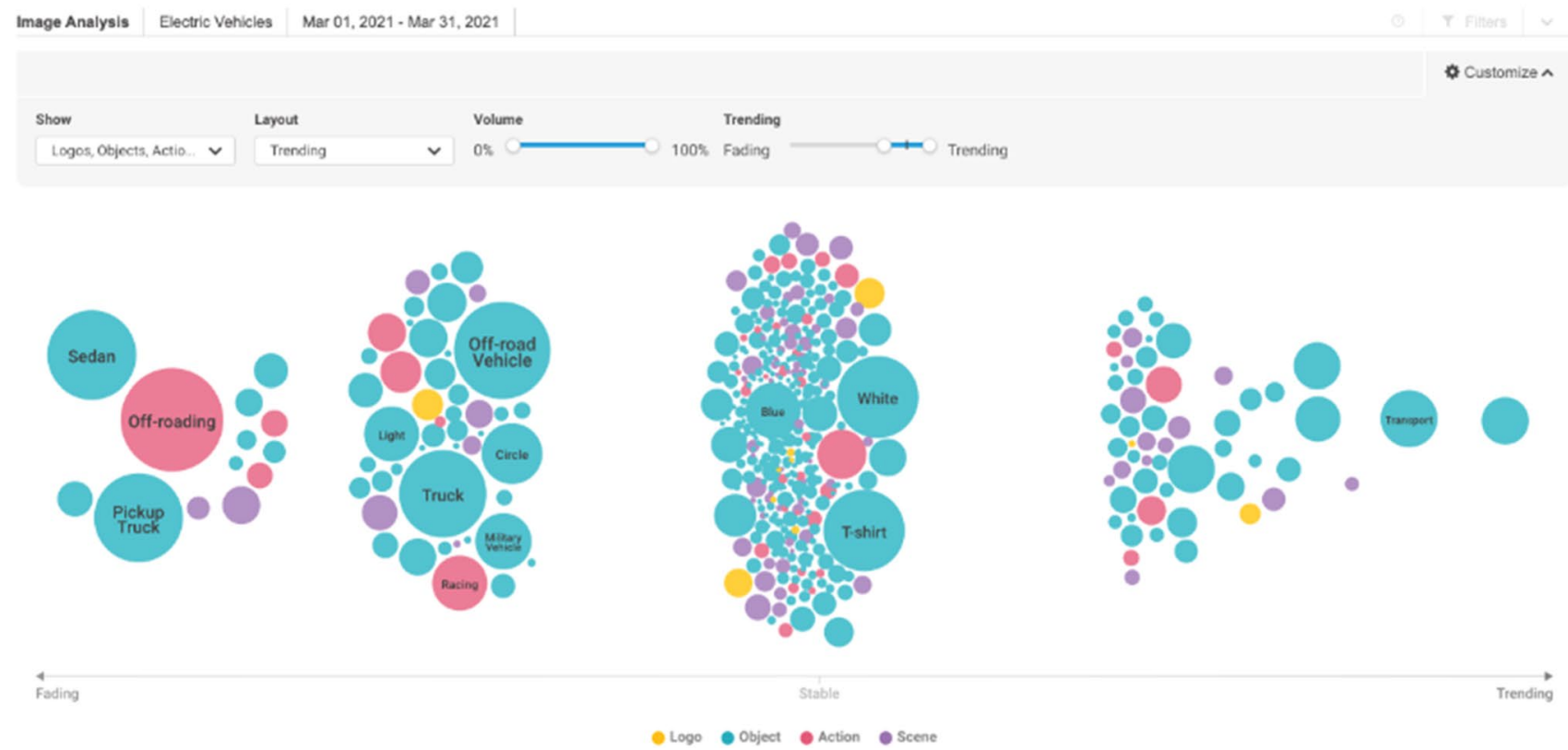

Fig. 7 Sample Brandwatch image analysis trends by image type 
used to meet business objectives and improve performance.

- "Techie" (technical/analytical) skills that enable the collection and analysis of the data using various data management and analytical techniques.

- "Data designer" skills, such as data storytelling, that help technical and non-technical audiences easily consume the analysis results and apply the analytical insights.

The thoughtful integration of SLPs into marketing analytics course designs can accelerate the development of these in-demand skills.

Given this increase in demand for multi-disciplined marketing analysts and the rapid, ongoing change that exists in the digital marketing landscape, it is important that the course design leverages academic and practitioner insights (Liu and Burns 2018; Reibstein et al. 2009). The course design should "balance theoretical concepts and the development of practical skills" (Kim 2019). The course should also integrate topics in both social media data analysis and marketing strategy development (Kim 2019). This will help marketing students to further develop data-driven marketing skills that allow them to effectively use social media insights to solve present day marketing problems. This is an area that students have "lacked training" in the past (Crittenden and Crittenden 2015; Duffy and Ney 2015). It is also critical to implement a hands-on, "learning-by-doing" approach that utilizes context-relevant business problems and data (Lim and Heinrichs 2021; Chen et al. 2012). Finally, it is important to "elevate the purpose of analytics and marketing beyond employability" (LeClair 2018). For example, "democratizing data" to build a culture of informed decision-making or applying analytic-driven, customercentric insights to create greater "customer value" are two worthy goals that should inspire marketing students to hone both their marketing and analytical skills (LeClair 2018).

\section{Adopting a project-based learning methodology}

The discovery, tracking, preparation, analysis, and deployment steps were previously introduced as a comprehensive framework for performing social media analytics. Therefore, it is important to utilize a learning methodology that coincides with and complements these phases of SMA.

Also, given the hands-on nature, and the need to bridge the art and science of marketing, an experiential, projectbased learning (PjBL) approach is recommended when teaching social media analytics. PjBL is commonly used in education and is particularly relevant in the design of business and marketing analytics courses. It is defined as an "activity-based methodology, engaging students to perform activities involving research, decision-making, and writing" (Yazici 2020). Group work and learning through collaboration with team members are also a critical element of the PjBL approach (Yazici 2020).

The following four PjBL learning phases (Yazici 2020) are adopted as a relevant model for teaching social media analytics in marketing analytics courses (see Fig. 8).

\section{Hypothetical classroom project and objectives}

The following summary of suggested steps across each learning phase will describe how to introduce and utilize various features of the Brandwatch for Classrooms product in a classroom assignment. However, please keep in mind that other social listening platforms have similar features and it would be possible to substitute a different SLP into this learning framework.

Also, the suggestions are based on developing a project that focuses on the Brand Management and Competitor Analysis use cases. More specifically the primary objective of the project is to conduct a thorough analysis of a brand and its top competitors using the vast amount of social media data that can be extracted and analyzed using the Brandwatch platform. Students are then expected to use the brand and competitor analysis insights to develop relevant,

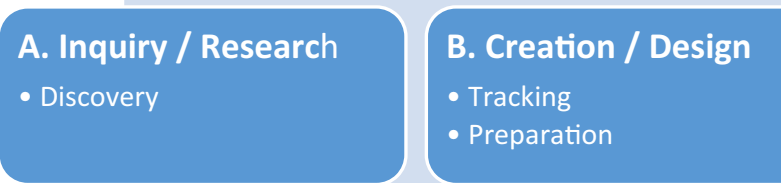

C. Analysis /

Implementation

- Analysis
D. Interpretation /

Evaluation

- Deployment

Fig. 8 Project-based learning methodology mapped to core SMA process steps

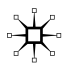


data-driven, marketing recommendations. It is possible to utilize this same learning methodology for different project objectives and use cases such as campaign measurement, market research, or customer experience management.

\section{Inquiry / research learning phase}

This introductory phase will require students to gain a rapid orientation and understanding of the social listening platform. The Brandwatch for Classrooms license includes an abundance of training videos that students should review to get an initial understanding of the core product features. It is also recommended that students complete the Brandwatch Foundations exam. This will test students' understanding of the platform, and, if students get an exam score of 80 or above, they receive the Brandwatch Foundations Certification which can be posted on their LinkedIn accounts (Brandwatch 20210).

Student groups should also be created as the project and student experience will be enhanced through regular peer-topeer and group-to-instructor collaboration. This also closely resembles the approach employees would use to perform SMA in the workplace.

The primary objective of this phase, given it utilizes an experiential learning approach, is to have student groups select a brand and associated competitors (possibly top 2 competitors) that will be studied in the social media analysis. Students will also use initial high-level insights gathered in this phase to begin to formulate a set of project questions and hypotheses that will guide the design of the subsequent analysis plan (Yazici 2020).

Students can utilize the Brandwatch Search feature which provides an easy way to perform initial research on candidate companies for the project without writing comprehensive queries.

Instructors should review a variety of use cases for social media analytics to help students get a clear understanding of how the insights are often applied by organizations. Also, time should be devoted teaching students the art of crafting empirical questions associated with different use cases and business objectives. (Siegel 2021). For example, a few empirical questions related to a competitor analysis use case could be subsequently answered using the SLP are:

- What types of audience segments are engaging with the competitor brands?

- Is the makeup of the engaged competitor audiences changing over time?

- Are the profile of competitor audiences significantly different than my brand's target audience?

\section{Creation / design learning phase}

The primary goals in this second learning phase are to have the students design and create the analysis plan, perform data acquisition, and prepare the data for analysis (Yazici 2020).

The analysis plan should describe how the empirical questions and hypotheses compiled in the prior phase will be analyzed and answered. The plan should include the type of social media data to be collected, the audience to be analyzed, the time period(s) to studied, the data categories/segments that need to be created to properly answer the analysis questions.

Students will use the Brandwatch Queries tool to write the Boolean logic that extracts the information to be used in the analysis. Students should be expected to test the precision of their query by analyzing a representative sample of the social media mentions that are extracted. This is an iterative process, switching between testing and query logic refinement. Also, students should leverage the image analytics capabilities of the platform and include brand logos and/ or image content in their query logic.

Students will also prepare the data for analysis in this phase. This will most likely include the development of new categories using the Brandwatch Categories and Rules features. For example, students may want to create a category dimension that can be used later in the analysis phase that includes all of the product categories sold by the brands selected for the study.

Students should also be challenged to train a machine learning (ML) model to create a category dimension that would be difficult to categorize with simple keyword rules. For example, categorizing consumer mentions into different stages of the customer buying lifecycle would be difficult by using only selected keywords. Instead students can use the Brandwatch Custom Classifier ML tool to train a model to accurately classify mentions into the appropriate customer lifecycles. Figure 9 (labeled Fig. 2 in the Forrester Research report) highlights typical marketing objectives, tactics, and measurement metrics at each stage of the lifecycle (Liu 2021). When students complete their SMA analysis, they should be asked to revisit these tactics and use their lifecycle-level SMA insights to propose insight-driven, personalized, social marketing tactics at each stage.

It is important to include readings and lectures on topics such as NLP (Hair Jr. et al. 2021) and image analytics (Shin et al. 2020) during this learning phase to provide a deeper understanding of the concepts and theories underlying these emerging technologies and their applications in a marketing environment. 
Fig. 9 Social marketing objectives follow the customer lifecycle

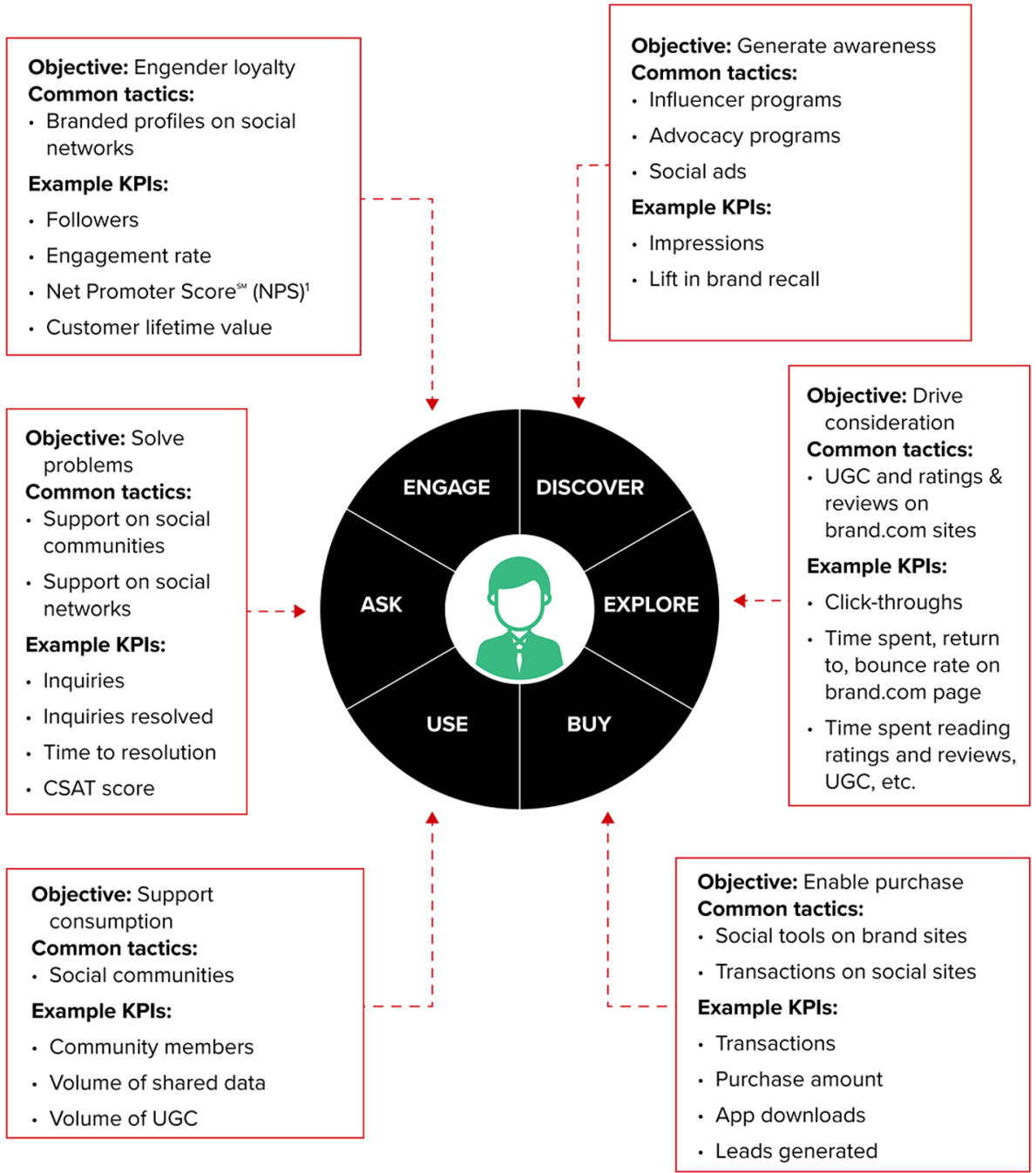

\section{Analysis / implementation learning phase}

The primary objective of the third learning phase is to perform a comprehensive data analysis that will likely include at a minimum data visualizations, descriptive statistics, audience segmentation, benchmarking, and trend analyses.

Students should use multiple Brandwatch Dashboard templates to conduct the comprehensive SMA analysis. For example, the analysis plan could include the following components (Jamali 2020):

- Share of voice analysis (volume of mentions)

- Customer sentiment analysis (distribution of positive, neutral, and negative mentions)

- Trend analysis (summary of various metrics across time periods)

- Audience analysis (profile of target segments by demographics, location, interests, and professions)

- Image analysis (summary of content appearing in the image data)
- Content source analysis (analysis of most popular content channels/sources)

- Topic modeling (summary of most popular, trending, and fading topics)

- Influencer analysis (summary of top authors and corresponding reach and impact scores)

What makes the analyses listed above even more meaningful and capable of answering the empirical questions compiled in the inquiry/research learning phase is the utilization of the category dimensions built in the creation/ design learning phase. For example, comparing the share of voice by each of the brands in the study is possible if students have built a brand category dimension. Also, evaluating customer sentiment for each brand, across each stage of the customer lifecycle, is possible if students have built customer lifecycle and brand category dimensions.

During this phase, instructors should demonstrate how to use the social listening platform to perform multi-dimensional analyses and explain how these can be crafted to 
directly answer the empirical questions established in the analysis plan.

\section{Interpretation / evaluation learning phase}

The primary objective of the final learning phase is to interpret the analysis results, clearly communicate the results to a diverse audience, and translate the knowledge into actionable business recommendations (Yazici 2020; Jaggia et al. 2020).

This final phase emphasizes the development of data storytelling skills. "Data storytelling refers to crafting and delivering compelling data-driven stories to decision makers for the purpose of converting insights into actions" (Jaggia et al. 2020). This requires the development of complementary skills in data analysis, data visualization, and the development of a compelling narrative (Jaggia et al. 2020). The data visualization should incorporate contrast such as size, color, and shape to make it easier for the audience to interpret and consume the information. The narrative should "ensure clear meaning" through the use of concise, unambiguous headlines, descriptive subtitles that clearly summarize the key insight(s), and visual cues and annotations that direct the audience to important data points (Hartman 2020).

Students can use the Brandwatch Report features that enable the export of dashboard visualizations and descriptive statistics. However, students should be challenged to create a complete report and supporting presentation that includes all of the data storytelling elements described above.

Instructors should incorporate readings, lectures, and exercises that clearly demonstrate the process and the steps involved in developing compelling stories with data.

This type of experiential project also provides an excellent opportunity to discuss possible ethical issues related to consumer data collection and the use of analytics. Social listening platforms showcase the breadth of data that can be captured on consumers. Used appropriately, this data can provide positive outcomes for consumers and businesses, by fueling customer-centric product innovation that drives business growth. However, if it is misused, intentionally or unintentionally, it can have harmful results.

For example, what are the reasonable bounds of using social media data and analytics in an attempt to influence behaviors? (LeClair 2018). The Facebook/Cambridge Analytica scandal that was exposed in 2018 is an example of a data breach that is estimated to have affected 50 million Facebook users that did not know their profile data was harvested to support targeted political campaigns (Confessore 2018). Also, how can we ensure that the data collected are not biased, potentially leading to misleading analysis findings and misguided applications of the insights (Hair Jr. et al. 2021)? These are critical issues, and it is appropriate to challenge students to regularly reflect on the appropriate and inappropriate use of consumer data. It is also an opportunity for the instructor to inspire and empower students to use ethical and socially responsible data collection and analytic practices when they enter the workforce.

\section{Brief statement on classroom project assessment}

Project-based learning approaches often adopt a project management assessment methodology. That is, individual students and student group progress is evaluated using a variety of assessment metrics at each of the four learning phases, and then a full project assessment is incorporated at the completion of the project (Yazici 2020).

The project described in this article provides the opportunity for student assessment across many important skill development areas such as: problem solving, critical thinking, logical query development, analysis and data synthesis, data storytelling and communication, oral presentations, and teamwork/collaboration (Yazici 2020).

\section{Conclusion}

Usage of social media platforms continues to increase at a rapid pace with over $50 \%$ of the global population actively using one or more platforms for commerce, socializing, entertainment, education, and more (DataReportal 2021). A brief summary of 2020 per minute engagement rates on selected social media platforms illustrates the incredible scale of social media and online usage: 347,222 Instagram users post stories every minute, 147,000 Facebook users upload photos (per minute), 138,889 Instagram users click business ads (per minute), YouTube users upload $500 \mathrm{~h}$ of video (per minute), 69,444 LinkedIn users apply for jobs (per minute), consumers spend $\$ 1,000,000$ online (per minute), and Amazon ships 6659 packages every minute (Domo 2020).

Given this extreme level of consumer activity on social media platforms that was only heightened during the COVID-19 pandemic, it is not surprising that the business community is aggressively looking for opportunities to mine social media data and use the insights to inform business and marketing decision making. Many businesses are investing in social media analysts and social listening platforms (SLP) to support their data collection and analytics needs.

These rapid advancements in marketing practices and analytic technologies create significant ongoing challenges for educators and marketing researchers that require "greater multidisciplinary collaborations across the marketing, information technology, and strategy domains" (Grewal et al. 2020). The Brandwatch for Classrooms license agreement gives educational institutions the ability to integrate the Brandwatch Consumer Research platform 
into relevant courses such as marketing, analytics, social media, market research, branding, and business strategy at a compelling price point. For educators seeking opportunities to enrich their courses with additional experiential learning experiences, the Brandwatch platform provides students with real-time access to conversations on any topic, brand, or person they wish to research using data from over 100 million different sites (Brandwatch 2021p).

The Brandwatch platform gives educators and students access to a comprehensive suite of tools to perform each phase (discovery, tracking, preparation, analysis, deployment) of social media analytics. This is a key consideration when selecting a SLP to use in the classroom. Educators have limited time in a semester to introduce and train students on new analytic tools; therefore, the selection of a SLP that can perform all SMA tasks provides efficiencies, requiring shorter training ramp-up times compared to a multi-tool approach to performing social media analytics.

Another important consideration is the availability of platform documentation and training resources. Students should be able to use a self-directed approach for platform onboarding and training. This requires access to training videos, detailed product/feature documentation, exams and exercises to assess student knowledge, and wellwritten case studies to showcase how social media analytical insights can be applied across different use cases. The Brandwatch Help Center provides access to each of these resources and offers a professional certification that is highly valued by students and employers.

Beyond the technological challenges, teaching topics in social media analytics are subject to fast-pace change that also present pedagogical challenges. The expectation from students and the business community are applicationfocused courses that emphasize the development of technical skills over marketing theory (Muñoz and Wood 2015). It is critical to include a "theoretical and practical balance" in the course design. Developing technical skills is important, but it is equally important to integrate marketing concepts and theories into the course design (Duffy and Ney 2015; Muñoz and Wood 2015). The ultimate goal is to develop "transferable skills" that allow students to apply the marketing concepts, regardless of the specific analytic platform they utilized in the classroom (Kim 2019).

The four phase project-based learning methodology described in this article is designed with this theoretical and practical balance in mind. The technology enables the efficient completion of the social media analytic steps, however the higher-level learning objective is to teach students how to confidently and comprehensively use social media data to develop insight-driven, customercentric, marketing strategies that are informed by the selfidentified needs and preferences of consumers (Kim 2019).

\section{References}

Alp, Z.Z., and ŞG. Öğüdücü. 2018. Identifying topical influencers on twitter based on user behavior and network topology. Knowledge-Based Systems 141: 211-221.

Bal, A.S., D. Grewal, A. Mills, and G. Ottley. 2015. Engaging students with social media. Journal of Marketing Education 37 (1): 190-203.

Brandwatch. 2021a. Brandwatch use cases. https://www.brandwatch. com/use-cases/.

Brandwatch. 2021b. Brandwatch for classrooms. https://www.brand watch.com/p/brandwatch-for-classrooms/.

Brandwatch. 2021c. Brandwatch Consumer Research: sample use interface images. Brandwatch Product Marketing team.

Brandwatch. 2021d. Brandwatch Consumer Research: Product terminology. Brandwatch Help Center. https://consumer-researchhelp.brandwatch.com/hc/en-us/articles/360013467157-Produ ct-Terminology (license required to access web page).

Brandwatch. 2021e. Brandwatch Consumer Research: Brandwatch Search. Brandwatch Help Center. https://consumer-researchhelp.brandwatch.com/hc/en-us/articles/360013596217-Brand watch-Search (license required to access web page).

Brandwatch. 2021f. Brandwatch Consumer Research: What is AIbased search? Brandwatch Help Center. https://consumerresearch-help.brandwatch.com/hc/en-us/articles/3600197542 18-What-is-AI-based-Search= (license required to access web page).

Brandwatch. 2021g. Brandwatch Consumer Research: The query editor. Brandwatch Help Center. https://consumer-research-help. brandwatch.com/hc/en-us/articles/360012849298-The-QueryEditor (license required to access web page).

Brandwatch. 2021h. Brandwatch 2021 Roadmap.

Brandwatch. 2021i. Brandwatch Consumer Research: Social panels. Brandwatch Help Center. https://consumer-research-help. brandwatch.com/hc/en-us/articles/360013614718-Social-Panels (license required to access web page).

Brandwatch. 2021j. Brandwatch Consumer Research: Custom classifiers. Brandwatch Help Center. https://consumer-research-help. brandwatch.com/hc/en-us/articles/360012853738-Custom-Class ifiers (license required to access web page).

Brandwatch. 2021k. Brandwatch Consumer Research: Dashboard overview. Brandwatch Help Center. https://consumer-researchhelp.brandwatch.com/hc/en-us/articles/360012758457-Dashb oards-Overview (license required to access web page).

Brandwatch. 20211. Brandwatch Consumer Research: Influencers dashboard. Brandwatch Help Center. https://consumer-resea rch-help.brandwatch.com/hc/en-us/articles/360013683818-Influ encers-Dashboard (license required to access web page).

Brandwatch. 2021m. Brandwatch Consumer Research: Automated reports. Brandwatch Help Center. https://consumer-researchhelp.brandwatch.com/hc/en-us/articles/360013577337-Autom ated-Reports (license required to access web page).

Brandwatch. 2021n. Brandwatch Consumer Research: Custom alerts. Brandwatch Help Center. https://consumer-research-help.brand watch.com/hc/en-us/articles/360013577077-Custom-Alerts (license required to access web page).

Brandwatch. 2021o. Brandwatch Consumer Research: Foundation certification. Learning Zone. https://learning-zone-help.brand watch.com/hc/en-us/articles/360013526258 (license required to access web page).

Brandwatch. 2021p. Brandwatch Consumer Research: Data coverage. Brandwatch Help Center. https://consumer-research-help. brandwatch.com/hc/en-us/sections/360003575798-Data-Cover age (license required to access web page). 
Calheiros, A.C., S. Moro, and P. Rita. 2017. Sentiment classification of consumer-generated online reviews using topic modeling. Journal of Hospitality Marketing \& Management 26 (7): 675-693.

Chen, H., R.H. Chiang, and V.C. Storey. 2012. Business intelligence and analytics: From big data to big impact. Management Information Systems Quarterly 36 (4): 1165-1188.

Choi, J., J. Yoon, J. Chung, B.Y. Coh, and J.M. Lee. 2020. Social media analytics and business intelligence research: A systematic review. Information Processing and Management. https://doi.org/ 10.1016/j.ipm.2020.102279.

Confessore, N. 2018. Cambridge Analytica and Facebook: The scandal and the fallout so far. The New York Times. April 4, 2018. https:// www.nytimes.com/2018/04/04/us/politics/cambridge-analyticascandal-fallout.html

Crittenden, V., and W. Crittenden. 2015. Digital and social media marketing in business education: Implications for the marketing curriculum. Journal of Marketing Education 37 (2): 71-75.

DataReportal. 2021. Digital 2021 April Global Statshot Report: The latest insights into how people around the world use the internet, social media, mobile devices, and ecommerce. Kepios Pte.Ltd., We Are Social Ltd., and Hootsuite Inc., April, 2021. https://datar eportal.com

Domo. 2020. Data Never Sleeps 8.0: Infographic. Domo, Inc. August, 2020. https://www.domo.com/learn/infographic/ data-never-sleeps-8.

Duffy, K., and J. Ney. 2015. Exploring the divides among students, educators, and practitioners in the use of digital media as a pedagogical tool. Journal of Marketing Education 37 (2): 104-113.

Felbermayr, A., and A. Nanopoulos. 2016. The role of emotions for the perceived usefulness in online customer reviews. Journal of Interactive Marketing 36: 60-76.

Gandomi, A., and M. Haider. 2015. Beyond the hype: Big data concepts, methods, and analytics. International Journal of Information Management 35 (2): 137-144.

Grewal, D., J. Hulland, P.K. Kopalle, and E. Karahanna. 2020. The future of technology and marketing: A multidisciplinary perspective. Journal of the Academy of Marketing Science 48 (1): 1-8. https://doi.org/10.1007/s11747-019-00711-4.

Guellil, I., and K. Boukhalfa. 2015. Social big data mining: A survey focused on opinion mining and sentiments analysis. 12th international symposium on programming and systems. ISPS 2015: 132-141.

Hair, J.F., Jr., Harrison, D.E., \& Ajjan, H. 2021. Chapters introduction to marketing analytics, natural language processing. In Essentials of marketing analytics, 1st ed., pp. 1-27 and pp. 335-371. New York, NY: McGraw Hill Education.

Hartman, K. 2020. The day the geeks took over \& storytelling with data. In Digital marketing analytics: In theory and in practice, 2nd ed., pp. 2-38 and pp. 161-216. Ostmen Bennettsbridge Publishing Services.

Ikeda, K., G. Hattori, C. Ono, H. Asoh, and T. Higashino. 2013. Twitter user profiling based on text and community mining for market analysis. Knowledge-Based Systems 51: 35-47.

Jaggia, S., A. Kelly, K. Lertwachara, and L. Chen. 2020. Applying the CRISP-DM framework for teaching business analytics. Decisions Sciences, Journal of Innovative Education 18 (4): 612-634.

Jamali, C. 2021. Digital consumer intelligence in practice: Data segmentation. Brandwatch Guides.

Khan, G.F. 2018. Understanding social media analytics. In Creating value with social media analytics: Managing, aligning, and mining social media test, networks, actions, location, apps, hyperlinks, multimedia, \& search engines data, 1st ed., pp. 91-124. Seattle, WA: CreateSpace.

Kim, Y. 2019. Developing a work-ready social media marketing analytics course: A model to cultivate data-driven and multiperspective strategy development skills. Decisions Sciences, Journal of Innovative Education 17 (2): 163-188.

Kumar, A., R. Bezawada, R. Rishika, R. Janakiraman, and P.K. Kannan. 2016. From social to sale: The effects of firm-generated content in social media on customer behavior. Journal of Marketing 80 (1): 7-25.

LeClair, D. 2018. Integrating business analytics in the marketing curriculum: Eight recommendations. Marketing Education Review 28 (1): 6-13.

Lim, J.S., and J.H. Heinrichs. 2021. Developing context-relevant project experiences for marketing analytics students. Decisions Sciences, Journal of Innovative Education 19 (2): 150-156.

Liu, Jessica. 2021. Get smarter on social media measurement. Forrester Research Inc., May, 14, 2021.

Liu, Jessica, \& Dawson, Sarah. 2020. The Forrester wave ${ }^{\mathrm{TM}}$ : Social Listening Platforms, Q4 2020. Forrester Research Inc., November, 10, 2020.

Liu, Jessica, \& Dawson, Sarah. 2021. The Social Technology Convergence Stalls Out. Forrester Research Inc., June, 4, 2021.

Liu, Jessica, \& Parrish, Melissa. 2020. Marketers' options improve as social marketing technologies converge. Forrester Research Inc., July, 17, 2020.

Liu, X., and A. Burns. 2018. Designing a marketing analytics course for the digital age. Marketing Education Review 28 (1): 28-40.

Lynn, T., Healy, P., Kilroy, S., Hunt, G., van der Werff, L., Venkatagiri, S., \& Morrison, J. 2015. Towards a general research framework for social media research using big data. In 2015 IEEE international professional communication Conference (IPCC) 1-8.

McAfee, A., and E. Brynjolfsson. 2012. Big data: The management revolution. Harvard Business Review 90 (October): 60-68.

Miah, S.J., H.Q. Vu, J. Gammack, and M. McGrath. 2017. A big data analytics method for tourist behavior analysis. Information \& Management 54 (6): 771-785.

Moon, S., and W.A. Kamakura. 2017. A picture is worth a thousand words: Translating product reviews into a product positioning map. International Journal of Research in Marketing 34 (1): 265-285.

Muñoz, C.L., and N.T. Wood. 2015. Update status: The state of social media marketing curriculum. Journal of Marketing Education 37 (2): 88-103.

Nam, H., Y.V. Joshi, and P.K. Kannan. 2017. Harvesting brand information from social tags. Journal of Marketing 81 (4): 88-108.

Pääkkönen, J., S.M. Laaksonen, and M. Jauho. 2020. Credibility by automation: Expectations of future knowledge production in social media analytics. Convergence: the International Journal of Research into New Media Technologies 26 (4): 790-807.

Rehman, M.H., V. Chang, A. Batool, and T.Y. Wah. 2016. Big data reduction framework for value creation in sustainable enterprises. International Journal of Information Management 36 (6): 917-928.

Reibstein, D.J., G. Day, and J. Wind. 2009. Guest editorial: Is marketing academia losing its way. Journal of Marketing 73 (4): 1-3. https://doi.org/10.1509/jmkg.73.4.001.

SAS. 2020. Make every voice heard with natural language processing: Everything you need to know about communication between humans and machine intelligence, pp. 1-24. eBook. Cary, NC, SAS Press. https://www.sas.com/en/whitepapers/natural-langu age-processing-110641.html

Shin, D., S. He, G.M. Lee, A.B. Whinston, S. Cetintas, and K.C. Lee. 2020. Enhancing social media analysis with visual data analytics: A deep learning approach. MIS Quarterly 44 (4): 1459-1492.

Siegel, P. 2021. How to ask smart questions of online conversation data that matter to your business. Brandwatch Webinars.

Stieglitz, S., M. Mirbabaie, B. Ross, and C. Neuberger. 2018. Social media analytics-Challenges in topic discovery, data collection, 
and data preparation. International Journal of Information Management 39: 156-168.

Statista. 2021. Social media usage worldwide. GlobalWebIndex, We Are Social Ltd., DataReportal, Hootsuite Inc., January, 2021.

Wamba, S.F., S. Akter, H. Kang, M. Bhattacharya, and M. Upal. 2016. The Primer of Social Media Analytics. Journal of Organizational and End User Computing. https://doi.org/10.4018/JOEUC.20160 40101.

Wang, Y., Q. Deng, M. Rod, and S. Ji. 2021. A thematic exploration of social media analytics in marketing research and an agenda for future inquiry. Journal of Strategic Marketing 29 (6): 471-491.

Yazici, H.J. 2020. Project-based learning for teaching business analytics in the undergraduate curriculum. Decisions Sciences, Journal of Innovative Education 18 (4): 589-611.

Zeng, D., H. Chen, R. Lusch, and S.H. Li. 2010. Social media analytics and intelligence. IEEE Intelligent Systems 25 (6): 13-16.

Zhan, Y., R. Han, M. Tse, M.H. Ali, and J. Hu. 2021. A social media analytic framework for improving operations and service management: A study of the retail pharmacy industry. Technological Forecasting \& Social Change. https://doi.org/10.1016/j.techfore. 2020.120504 .
Publisher's Note Springer Nature remains neutral with regard to jurisdictional claims in published maps and institutional affiliations.

Mike McGuirk is a lecturer and full-time faculty member in the Marketing Division at Babson College, United States. Prior to joining Babson in January, 2020, Mike taught at Emerson College for four years, where he was a senior executive-in-residence and also the Graduate Program Director of the new Digital Marketing and Data Analytics master's program. Before transitioning to academia, Mike had been working in the analytics field for over 25 years as a data-driven marketing and analytics consultant. His research interests include business ethics, consumer data protection and privacy, customer analytics, marketing analytics, social media analytics, marketing strategy, and customer experience management. 